This trend remained the same for both viral and bacterial URTIs. Patients from less deprived households were less likely to be discharged and more likely to be admitted, for both short ( $<24$ hours) and longer admissions ( $>24$ hours). Mapping of patient postcodes highlighted 'hotspots' of patients in the North East.

Conclusion There is a significant relationship between social deprivation and presentation to Paediatric A\&E with URTI in the North East. Most patients presenting to Great North Children's Hospital Paediatric A\&E with URTI came from more socially deprived backgrounds.

\section{G220(P) THE ROLE OF ULTRASOUND AND PAEDIATRIC APPENDICITIS SCORE IN THE DIAGNOSIS OF APPENDICITIS IN CHILDREN}

${ }^{1} \mathrm{~K}$ Gudka, ${ }^{2} \mathrm{R}$ Khurram, ${ }^{3} \mathrm{H}$ Halim, ${ }^{4} \mathrm{~S}$ Hagh, ${ }^{5}$ I Suliman. 'Paediatrics, The Royal Free London NHS FT, London, UK; ${ }^{2}$ Acute Medicine, The Royal Free London NHS FT, London, UK; ${ }^{3}$ Hepatology, The Royal Free London NHS FT, London, UKi ${ }^{4}$ General Surgery, The Royal Free London NHS FT, London, UK; ${ }^{5}$ Colorectal and General Surgery, The Royal Free London NHS FT, London, UK

\subsection{6/archdischild-2020-rcpch.188}

Aims Appendicitis is the most common abdominal surgical emergency in children. However, the diagnosis can often prove to be challenging. The aim of this study was to evaluate the role of ultrasound and clinical scoring tools such as paediatric appendicitis score (PAS) in facilitating the diagnosis of appendicitis in children who underwent an emergency appendicectomy.

Methods We retrospectively analysed the clinical records and preoperative imaging of a total of 39 patients under the age of 18 who underwent an emergency laparoscopic or open appendicectomy over a 6 month period between November 2018 and May 2019. Clinical history, examination findings and laboratory data were used to calculate PAS. The diagnostic performance of PAS and ultrasound were evaluated using histopathology as the gold standard to confirm the diagnosis of appendicitis.

Results Overall, there was a $13 \%$ negative appendicectomy rate. 20 appendicectomies (51\%) occurred in the high risk PAS group (PAS $\geq 7$ ), and this correlated with $100 \%$ positive histology for appendicitis. This compares with the intermediate risk PAS group (PAS 4-6) which had positive histology in $72 \%$ (13 children). 8 children (40\%) with a high PAS underwent an ultrasound preoperatively, compared to 18 children $(100 \%)$ with an intermediate PAS. When compared to the data from April - September 2018, there has been a 12\% reduction in use of ultrasound in high risk PAS $(52 \%$ to $40 \%)$. The was a delay of 11 hours in time taken to theatre between those children who had an ultrasound scan pre-operatively compared to those who did not.

Conclusions Despite there being some improvement, ultrasound is still being used extensively to investigate appendicitis in children. Overall PAS was a better tool than ultrasound in diagnosing appendicitis. This study has emphasised the importance of clinically risk stratifying patients into high, intermediate and low risk PAS. Ultrasound requests should be reduced in high risk PAS children due to delays in definitive treatment and a $0 \%$ negative appendicectomy rate. This in turn, should positively impact outcomes such as length of hospital admission, perioperative and postoperative complications.

\section{G221(P) A CHANGE IN THE APPROACH TO THE MANAGEMENT OF SORE THROAT IN CHILDREN}

'L Duthie, ${ }^{2} \mathrm{~L}$ Doan, ${ }^{2} \mathrm{~S}$ Griffiths, ${ }^{3} \mathrm{P}$ Munthali. 'Paediatric Intensive Care, Birmingham Children's Hospital, Birmingham, UK; ${ }^{2}$ Paediatrics, University Hospitals Coventry and Warwickshire, Coventry, UK; ${ }^{3}$ Microbiology, University Hospitals Coventry and Warwickshire, Coventry, UK

\subsection{6/archdischild-2020-rcpch.189}

Aims Sore throat and fever is a common reason for children to present acutely to the Emergency Department. Despite mainly being a self-limiting illness, antibiotics are often prescribed despite limited evidence of benefit. Previous practice had been to use bacterial throat swabs to identify children who require antibiotic treatment. However, recent NICE guidelines have promoted a score based approach to treatment (FeverPain or Centor) in order to limit antibiotic use and reduce resistance. This approach is still not followed uniformly, and in some units bacterial throat swabs are being taken routinely to guide treatment.

Our aim was to identify whether the use of bacterial throat swabs in our unit was leading to over-prescribing of antibiotics and the medicalisation of a primarily self-resolving illness

Methods We retrospectively reviewed the use of antibiotics for sore throat on 60 patients who had throat swabs taken between October and November 2018. Swab results were retrieved from the microbiology information system and FeverPain (FPS) score was calculated from clinical notes.

Results Clinical scores were not documented for any patients.

38 children (63\%) were prescribed antibiotics, 20 (53\%) of which were prescribed for 5 days and then discontinued after a negative throat swab result was received. Only 5 (8\%) patients were confirmed as having a Group A Streptococcus on their throat swab, 4 of whom had been initiated on antibiotics.

Of the 38 children given antibiotics, 18 (47\%) met the FPS criteria for treatment. (score 4 or 5). A further 6 out of the 11 children who were not prescribed antibiotics, also met the criteria.

Conclusions Less than half of those children who were treated with antibiotics would have required treatment if the FeverPain score was used. Implementing a score based approach would have reduced the overall antibiotic prescribing from $63 \%$ to $40 \%$. Not only would this reduce unnecessary antibiotic use and the costs associated with performing throat swabs but would also be more in line with practice in primary care, emphasising the self-limiting nature of the illness and the ability to manage at home.

\section{G222(P) FAMILY-CENTRED CARE PRIOR TO TRANSFER OF THE CRITICALLY ILL CHILD}

R Kirk, A Gite. Lister Hospital, East and North Herts NHS Trust, Stevenage, UK

\subsection{6/archdischild-2020-rcpch.190}

Background Managing and stabilising a critically ill child within a district general hospital prior to transfer to PICU can present communication challenges. Our study aims to identify ways in which teams can improve the delivery of family-centred care during this difficult time.

Methods We reviewed the notes of 14 children who were transferred from the resuscitation area of a district general hospital to a tertiary PICU over a one-year period. We then 